\title{
Making connections
}

\author{
Nature Ecology \& Evolution's broad scope brings together all strands of the story of biodiversity, and how our species
} fits in this ongoing narrative.

W hen we launched Nature Ecology \& Evolution 18 months ago, we wanted the journal to cover ecology and evolution research in the broadest possible sense, while making biodiversity and its relationship with humanity an important focus. We have been thrilled by how the quality and the breadth of the content we have published have allowed us to fulfil the mission we set out in our opening Editorial, and the contents of this issue are a good place to celebrate this holistic view of the field.

The Comment by Bliege Bird and Nimmo puts people at the heart of conservation biology by arguing that place-based societies provide critical ecosystem functions that should not be ignored in efforts to reduce the harmful environmental effects of industrialized societies. It was particularly pleasing to see the excitement of one of the authors on Twitter about the fact the article had engaged scientists from other ecological and evolutionary disciplines with whom he does not usually interact. We have hoped from the start that the breadth and interconnectedness of our content would facilitate such interactions, and it is encouraging to see this in action.

The interaction between humans and nature is a strong theme in several other articles in this issue. Ecker et al. examine the environment of Early Pleistocene hominins in southern Africa, showing that the distribution of $\mathrm{C} 3$ and $\mathrm{C} 4$ plants was quite distinct from that in eastern Africa, thereby adding nuance to how we interpret the effect of the environment on the early evolution of our species. In this respect, of course, there is nothing particularly special about humans. A palaeoecological study like this considers environmental effects on the evolution of a species in a way that is similar to that in equally important studies on stickleback adaptive radiation (Marques et al.), cavefish developmental biology

(Gore et al.) or avian biogeography and speciation (Pigot et al. and McEntee et al.).
Looking more recently in human evolutionary history, GaudzinskiWindheuser et al. present evidence for close-range spear hunting by Neanderthals, which probably required co-ordinated group behaviour. This type of interaction with other species is predation aided by technology, but a more profound change in our environmental interactions occurred when we domesticated other species. Wu et al. use genomics to examine the complex patterns of introgression that occurred between species of the Bos complex during domestication.

Compared with these older interactions, the interaction of modern industrialized human societies with nature is widely known and discussed. For example, Mazor et al. examine the landscape of research on the drivers of biodiversity loss, and interestingly suggest a mismatch between the impact of those drivers and the research effort on them. And on a more positive note, Shaver et al. show how local management decisions can improve the resilience of coral reefs in the face of climate change. However, there are aspects of modern society's environmental impact that are less well considered. In a Perspective article, Giraudeau et al. argue that humans are a cause of cancer in many wild animal species, and that this is not just through the obvious mechanism of chemical pollution but also via accidental and deliberate feeding, reduction in genetic diversity, and light pollution. The authors argue that the many well-known ways in which anthropogenic factors are carcinogenic for humans apply to wild animals too.

Environmental change is also affecting human health in multifaceted ways. Magid et al. examine the differential effect on adult male testosterone levels of having spent childhood in either the United Kingdom or Bangladesh: the UK environment, with a probable lower infectious-disease burden, leads to higher levels of testosterone. As Blackwell notes in the accompanying News \& Views, the conditions of high sanitation and nutrition currently available to millions of people may differ from those experienced at any previous point in human evolution, and could help explain changes in life history such as advanced onset of puberty, and changes in health such as increases in the rate of prostate cancer. Considering these hormonal differences from an ecological and evolutionary perspective is directly relevant to understanding the medical implications.

In this way, the scope of the journal is a continuum on which human-environment interactions and medical and social topics sit side-by-side with traditional fundamental topics in ecology and evolutionary biology. This issue showcases our enthusiasm for the latter as well, such as the evolution of cell types (Sebé-Pedrós et al.), mating types (Constable and Kokko), novel gene function (Jerlström Hultqvist et al.) and division of labour (Cooper and West); the environmental coupling of heritability and selection (Ramakers et al.); and the ecological role of leguminous plants in forest succession (Gei et al.).

This is also a good opportunity to mention that the journal's editors are regularly at conferences engaging with these varied but overlapping research communities. In the next couple of months, you will find us at the Society for Molecular Biology \& Evolution, the Gordon Research Conference on Ocean Global Change Biology, the European Evo-Devo meeting, the European Society for Human Evolution, the Ecological Society of America and Evolution 2018. For those not attending such conferences, the Nature Research Ecology \& Evolution Community is a forum for discussion of both published papers and more general topics of interest. We hope to see many of you at the conferences or on the community site, and look forward to wideranging discussions and making connections between different research niches.

Published online: 25 June 2018 https://doi.org/10.1038/s41559-018-0604-5 